\title{
Erratum: Model independent evaluation of the Wilson coefficient of the Weinberg operator in QCD
}

\author{
Tomohiro Abe, ${ }^{a, b}$ Junji Hisano ${ }^{b, c, d}$ and Ryo Nagai ${ }^{e}$ \\ ${ }^{a}$ Institute for Advanced Research, Nagoya University, \\ Furo-cho Chikusa-ku, Nagoya, Aichi, 464-8602 Japan \\ ${ }^{b}$ Kobayashi-Maskawa Institute for the Origin of Particles and the Universe, Nagoya University, \\ Furo-cho Chikusa-ku, Nagoya, Aichi, 464-8602 Japan \\ ${ }^{c}$ Department of Physics, Nagoya University, \\ Furo-cho Chikusa-ku, Nagoya, Aichi, 464-8602, Japan \\ ${ }^{d}$ Kavli IPMU (WPI), UTIAS, University of Tokyo, \\ Kashiwa, Chiba 277-8584, Japan \\ ${ }^{e}$ Department of Physics, Tohoku University, \\ Sendai, Miyagi 980-8578, Japan \\ E-mail: abetomo@kmi.nagoya-u.ac.jp, hisano@eken.phys.nagoya-u.ac.jp, \\ nagai@tuhep.phys.tohoku.ac.jp
}

ERRATUM TO: JHEP03(2018)175

ArXiv EPrint: 1712.09503

We have found that a factor $1 / 2$ should be multiplied by eq. (4.1) of the paper when we use the same vertex twice. For instance, the Wilson coefficient of the Weinberg operator $\left(C_{G}\right)$ induced by

$$
\mathcal{L} \supset-\bar{\psi}_{A} g_{\bar{A} A S} \psi_{A} S,
$$

with $S^{*}=S$ and $g_{\bar{A} A S}=X_{\bar{A} A S}\left(s+\gamma_{5} a\right)$ is calculated as

$$
\begin{aligned}
C_{G}=- & \frac{1}{2} \frac{1}{(4 \pi)^{4}} 6 \operatorname{Im}\left(s a^{*}\right) m_{A}^{2} \\
& \times\left\{\left(X T_{A} T_{A} X^{\dagger}\right) f_{1}\left(m_{A}^{2}, m_{A}^{2}, m_{S}^{2}\right)+\left(X X^{\dagger} T_{A} T_{A}\right) f_{1}\left(m_{A}^{2}, m_{A}^{2}, m_{S}^{2}\right)\right. \\
& \left.+2\left(X T_{A} X^{\dagger} T_{A}\right)\left[f_{2}\left(m_{A}^{2}, m_{A}^{2}, m_{S}^{2}\right)\right]\right\},
\end{aligned}
$$

where $f_{1}$ and $f_{2}$ are defined in eqs. (3.28) and (3.29). We have checked that the eq. (2) is consistent with the results reported in refs. [1-3]. 
Open Access. This article is distributed under the terms of the Creative Commons Attribution License (CC-BY 4.0), which permits any use, distribution and reproduction in any medium, provided the original author(s) and source are credited.

\section{References}

[1] S. Weinberg, Larger Higgs Exchange Terms in the Neutron Electric Dipole Moment, Phys. Rev. Lett. 63 (1989) 2333 [INSPIRE].

[2] M. Jung and A. Pich, Electric Dipole Moments in Two-Higgs-Doublet Models, JHEP 04 (2014) 076 [arXiv: 1308.6283] [INSPIRE].

[3] J.R. Ellis, J.S. Lee and A. Pilaftsis, Electric Dipole Moments in the MSSM Reloaded, JHEP 10 (2008) 049 [arXiv: 0808.1819] [INSPIRE]. 